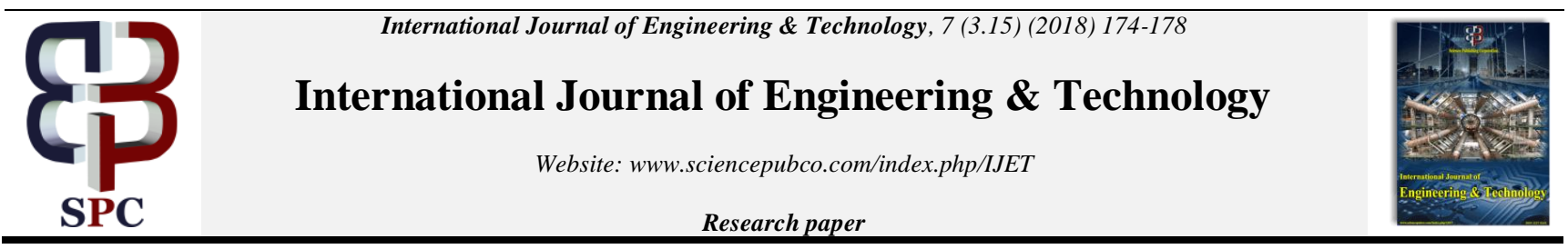

\title{
Real-Time Internet Based Attendance Using Face Recognition System
}

\author{
Yuslinda Wati Mohamad Yusof*, Muhammad Asyraf Mohd Nasir, Kama Azura Othman, \\ Saiful Izwan Suliman, Shahrani Shahbudin, Roslina Mohamad \\ Faculty of Electrical Engineering \\ Universiti Teknologi MARA \\ 40450 Shah Alam Selangor Malaysia \\ *Corresponding author E-mail:yuslinda@salam.uitm.edu.my
}

\begin{abstract}
This project focuses on face recognition implementation in creating fully automated attendance system with a cloud. Cloud services will provide a useful information regarding the attendance such as attendance summary performance and visualizing the data into graph and chart. In this study, we aim to create an online student attendance database, interfaced with a face recognition system based on raspberry pi 3 model B. A graphical user interface (GUI) will provide ease of use for data analysis on the attendance system. This work used open computer vision library and python for face recognition system combined with SFTP to establish connection to an internet server which runs on PHP and Node.js. The results showed that by interfacing a face recognition system with a server, a real-time attendance system can be built and be monitored remotely.
\end{abstract}

Keywords: Attendance; Face Recognition; Internet of Thing; Raspberry PI; Real Time.

\section{Introduction}

Recording of attendees to an event is important for any organizations either for corporate offices, activities in school, universities, industries and etc[1]. Attendance records should have details information about the attendees such as start and end time, date, and venue. A good attendance system must be able to record the presence of an individual avoiding the act of manipulating attendance for instance, asking somebody else to sign on that person behalf.

Traditionally, attendance recording involves the use of paperbased name list to be distributed among attendees or participants for a particular event. This process is somewhat time consuming and troublesome should the paper lost during the event. This method also allows for cheaters to manipulate the process like signing in for an individual that is actually absent from the event. A centralized and computerized attendance database is needed for evaluation and generation of proper attendance report. Currently, there are few existing attendance recordings using database locally by storing it in local hard disk. Thus, utilizing a centralized database with Internet of Things (IoT) for instance offers advantages in term of better data backup and recovery should failure occurs at the local storage center.

Recently, a lot of developments emerged to enhance ways of attendance recording using state-of-the-art technology, among others are using the RFID card, voice print and biometric identification [1-4]. However, each of the mentioned methods does have its pro and cons.

The latest in attendance recoding is by employing face recognition algorithm. This system uses the latest facial detection and recognition algorithm with video camera to capture live image or video stream. The face recognition is achieved using an algorithm called the haar-cascade which is simpler as compared to viola-jones method which will then combine with facial recognition algorithm like the Eigen faces and local binary pattern histogram [1].

GUI applications provide good system interaction between user and system provider. Besides that, the use of GUI benefits user for better data visualization in data representation. Hence, this work will focus on developing attendance system using face recognition, with front-end system using graphical user interface and the back-end system with database. It is expected that the developed system will improve the existing attendance recording.

The system is implementing an online database and a graphical user interface (GUI) interface with a face recognition system on a Raspberry Pi 3 based on open computer source vision (OpenCV) version 3.1.0. Face recognition data used in this study will be analysed using three algorithms in OpenCV which are Eigen face, Fisher face and Local Binary Pattern Histogram Algorithm. Data were sampled from 29 volunteers from class EE241C7A of Faculty of Electrical Engineering, Universiti Teknologi MARA, Shah Alam, Selangor, Malaysia. This project is limited to the program written in python that is only tested and usable on a Windows 7 machine and a raspberry pi 3 running Raspbian Jessie with python and required plugins installed. And thus the attendance analysis use in this study only covered the standard method and data used in standard attendance analysis to provide a useful information on the GUI.

\section{Literature Review}

The method of taking attendance manually using attendance sheet or so called paper-based are time consuming and full of flaws [2][4]. Manual attendance sheet that require student to pass it around is distracting the students and hence disturbing the lecture. Thus, in this decade there emerge various way and method of taking 
attendance such as using RFID, thumbprint, punch card, smartphone application and face recognition. All these methods have their own advantage and disadvantages. For example, RFID based attendance system would require direct contact of the person to update their attendance [5]-[7]. This method is not efficient for large classes as students need to queue in order to update their attendance in the system. Another method that has been introduce is to use mobile application with voice print to update the attendance as described by [8] and [9]. Then there is another method which require lecturer or professor to call out students names, that would give the similar result as the paper-based attendance which would be time consuming and causes delay to start on the class [8].

In a different way is by using mobile application and voice print is using the on geo-location to track the person to update the attendance [8]. However, problem may arise for such cases of mobile phone runs out of battery and that would cause some problem for the management and the student as well. Combining more than one method for attendance might be great for security, but it will cause a lot of expenses and make the attendance time consuming. For example, using both RFID and face recognition, it would be redundant for an attendance which to focus on attendance and does not require any security measure that would require such system [3].

There is also a method using a marking system which will be detected by a video camera. This method requires a person to wear some kind of marked onto their body. This is more suitable for a uniform-based attendance like soldiers, but not suitable for student or any casual events that require attendance [10]. Lastly, a method of using face recognition-based attendance system. There are various facial recognition algorithm available to use such as Violajones algorithm and Eigen faces algorithm [2], [4], [11]. These algorithms have their own advantage and disadvantage. For such, lighting conditions and human pose would affect the face detection. Despite that, both algorithms can be implemented using various programming language such as MATLAB, visual basic (VB.net) and $\mathrm{C}++$ have 97 percent of accuracy [11]. This particular face recognition method require almost no direct contact with the system, and only require faces to be detected from a certain pose for a few seconds in the camera and the attendance can be updated. All this various method for taking attendance have also be implemented with a web-based attendance but none of these research does really look inside the database [2], [5], [10]. More and more people are preferable towards an online attendance that far more suitable with today's advanced of technology [12]. A windows-based application such as EAMS is limited to a windows platform machine and would require user to have certain dot net plugin and require the same platform in order to edit or add data to the system [12].

\section{Methodology}

The design of the system can be divided into two main parts which are face recognition system and the server system. The server or back-end system can be further divided into smaller chunks including database design, real-time log file monitoring, graphical data analysis and front-end design. Fig. 1 shows the overall flow chart of the system. To ensure the network are not flooded with data transfer from a single system, an interval of 5 minutes is put in between data transfer although this can be adjusted as required Fig. 2 shows the system architecture. Because the output is accessed via a web page, it can be accessed from any device with an internet connection and a web.

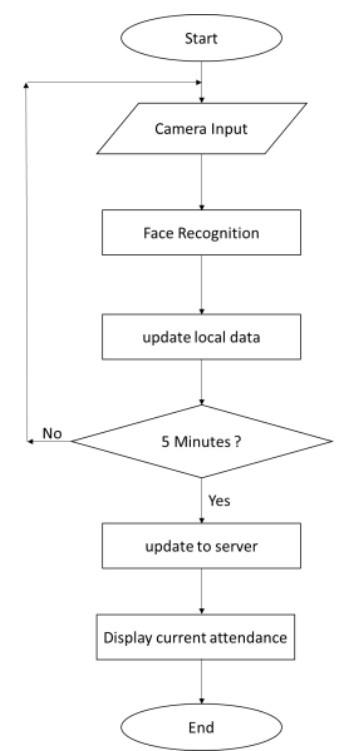

Fig. 1: Flow chart of the system

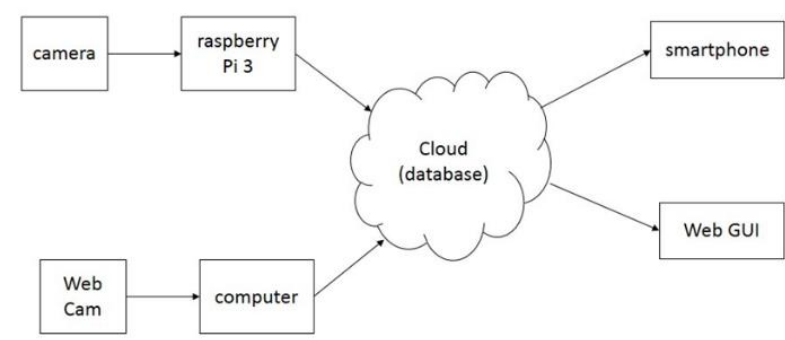

Fig. 2: Overall System Architecture

\subsection{Face Recognition System}

The face recognition is built on raspberry pi 3 model $\mathrm{B}+$ with a 5MP camera module. The system built using python which is a cross platform programming language and based on OpenCV library version 3.1.0. The face detection and recognition are using haar-cascade classifier for face detection and combined with local binary pattern histograms (LBPH) for face recognition. The systems are also designed with a graphical user interface to make sure that the back end system can be used properly without the need of intense training.

The face recognition system detects frontal face of a student and then compare it with face database in the system. If the student face matched, the monitor will show the student matrix number and update the attendance log file with a timestamp. When a face is detected the image undergo several processes before the image is save or compared with images in the database. Fig. 3 shows the face recognition flow chart. The image is first normalizing and then once again applied a histogram equalizer. First the image is stored in Blue Green Red (BGR) color model which is the reverse order of Red Green Blue (RGB) color model as in Fig. 4(a). Then the image is converted into LAB color model which stands for Luminance and A and B which is a chromatic component. Fig. 4(b) shows the image in LAB color model. These 3 components are then split into its own channel. The L channel is then equalizing by applying histogram equalization using Contrast Limited Adaptive Histogram Equalization (CLAHE) algorithm. This method will increase the contrast of the image as shown in Fig. 4(c). After CLAHE is applied to the L-channel, all three components are merge back into LAB color model. The image is then converted back into BGR model which then converted into gray color image. This gray image as in Fig. 4(d) are the final products of image normalization and then once again applied a normalize histogram to increase the image contrast. Fig. 4(e) shows the final product of the equalized gray image. 


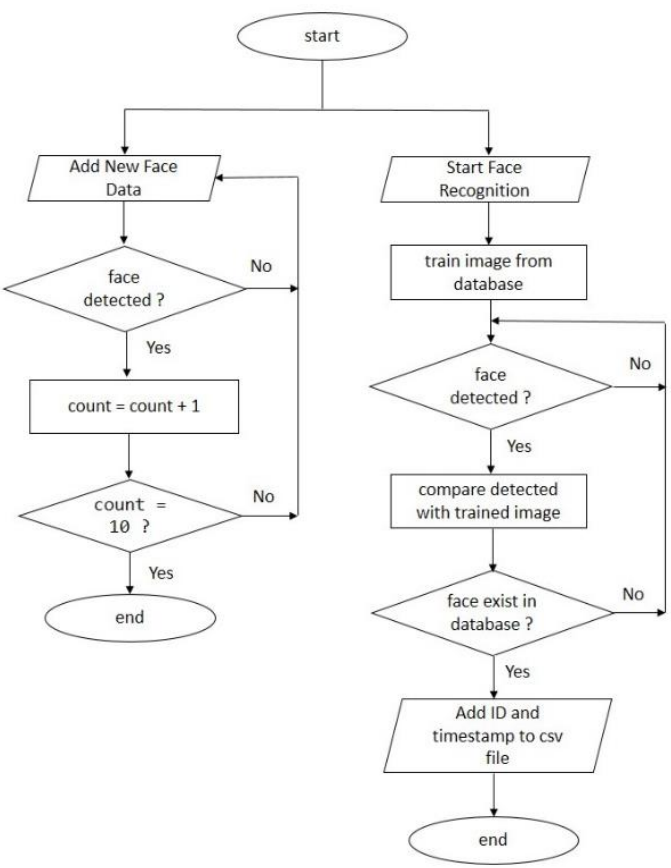

Fig. 3: Face recognition Process Flow

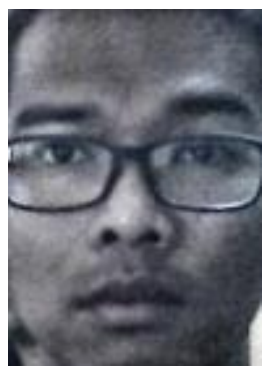

(a)

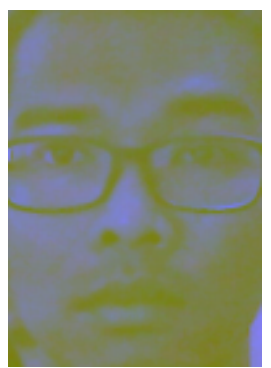

(c)

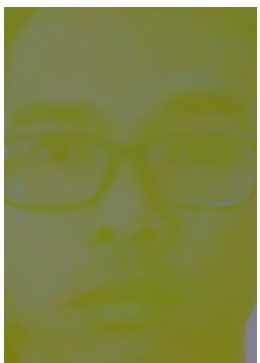

(b)

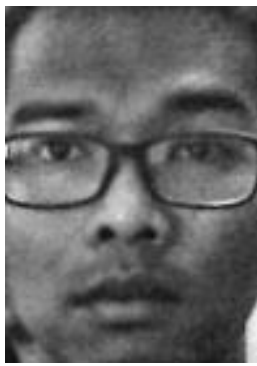

(d)

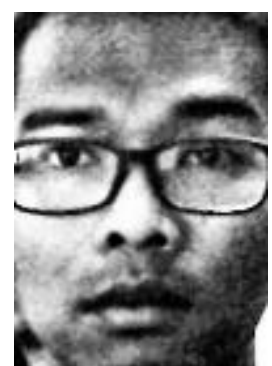

(e)

Fig. 4: Process to convert Image

\subsection{Real-Time Log File Monitoring}

Fig. 5 depicts the flow real-time file monitoring. The real-time log file monitoring is used to make sure that the database is update in real-time within 30 minutes when the class start. Any student who comes late more than 30 minutes are considered as absent automatically and the status can only be change directly by the lectur- er. In order to do this, PHP cannot be used because PHP is a scripting language which can only executed when a php page is requested by user. So node js which is an asynchronous serverside JavaScript runtime that runs on server side [13]. Using Node.js, it enable any changes on a file to be monitored and act upon as in Fig. 6. In this system, whenever the CSV log file change, the change will be update to the server database. This will make sure the data in the database is updated as the log file is sent from the face recognition system.

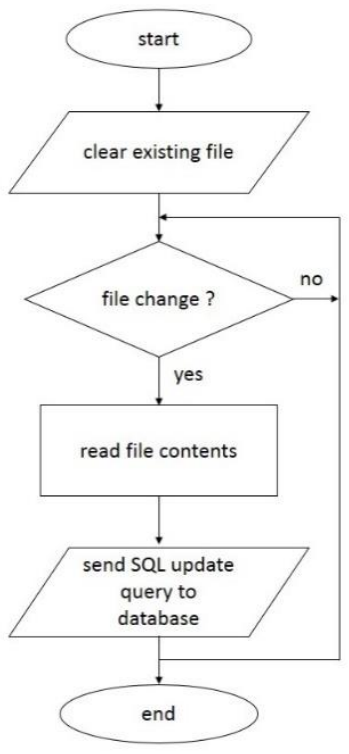

Fig. 5: Flow of Real Time

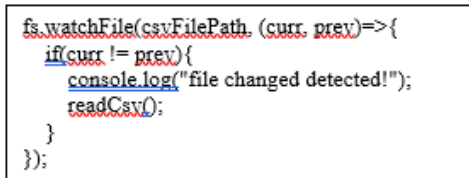

Fig. 6: File change detector

\subsection{Database Design}

Database is design according the Relational Database standards. This is design by designing a proper conceptual model and logical design consideration. This is to make sure that all information is located in its own table and are linked with each other without the needs to repeat the same data in all table. Database design is important because this is where all the data are stored and related to the front-end or the user-interface of the system as the data and user will interact a lot using PHP script.

\subsection{Data Consideration}

Graphs Data that are chosen to be shown on the dashboard are consider as the most important information that user will look for in a normal attendance sheet and represented in way that would it easier for user to understand the data. This data is calculated using data in the database and converted into percentage and are regularly updated to make sure a real-time data from the database is used.

\subsection{Front-End and Framework}

The front-end part is design considering two important factor in a user-interface design which are usability and user-experience. Usability means the user-interface is fully functional without bugs or hidden functions that require special skill while user-experience is the design concept that allow first-time user or inexperience user to navigate and use the interface easily without the needs of intensive manuals. Finally, the design also considers the looks of the user-interface so that it is design according to the design industry standard and practices. This is done by using design frame- 
work such as bootstrap which enable html elements to be manipulated easily with pre-configured cascading style sheet (CSS) styling.

After the layout is designed, a proper data representation is chosen to use a graphical representation which are in charts. This representation is done using Chart framework which is a JavaScript framework that enables char and graph to be easily generated.

\section{Results and Discussion}

The prototype has been simulated and tested for face recognition system, server back-end and internet front-end with a group consist of 29 students including 5 students with data already in the face database.

\subsection{Face Recognition System}

The face recognition system is set with a threshold value which will define the accuracy acceptance for the face recognition algorithm. A set of 10 data is taken for 5 students as the face database data. The sample data taken is $150 \times 150$ pixels each. This is due to the original size of image which is $1024 \times 720$ pixel. Fig. 7 shows the sample data taken from one student. When student walk in front of the camera, the system will detect the frontal face and compare it with the dataset from the face database. Student matrix number will be shown on the screen if the data match, otherwise it will display as unknown. Within the system, a comma separated value (CSV) file is generated with student matrix and timestamp of the detection. The output CSV file is verified to make it follow a proper CSV file format with a header as depicted in Fig. 8. The CSV file is re-created every time the system started. For every 10 seconds, the system will connect to the server using local area network (LAN) connection to upload the CSV log file using secure file transfer protocol (SFTP). and PYSFTP module.

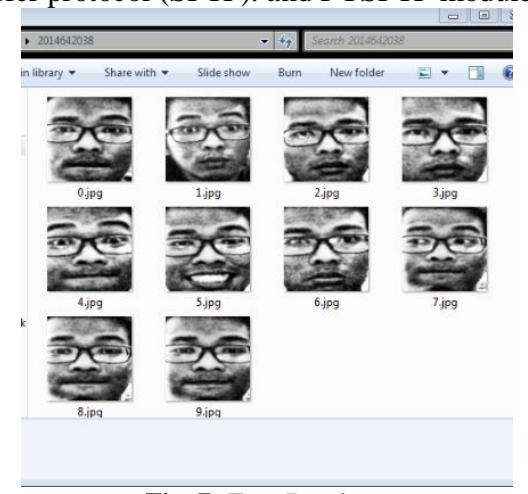

Fig. 7: Face Database

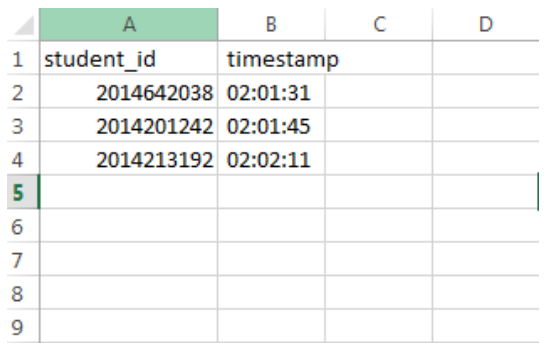

Fig. 8: Sample CSV log file generated

\subsection{Server Back-End}

The server act as a SFTP server, real-time file monitoring and a database. SFTP is used instead of FTP to make sure data integrity and establish a secure connection to the server. Local area network connection is used to make sure security of the system that no unauthorized user can access the database. SFTP server is created using Core SFTP Server software and the client are using python PYSFTP Module in order to use SFTP. Using SFTP has proven that the file can be transferred securely without error compare to normal file transfer protocol (FTP) which sometimes will result in file corruption and the file cannot be uploaded properly.

When the server receives the file from client which is the raspberry pi face recognition system, a node.js program (which run in real-time) will detect any changes every time the file is uploaded. Any updates on the file will be update to the MySQL database by the node js program in real-time. This is done using structured query language (SQL) query from the file change detector program to the database.

\subsection{Database and Data Analysis}

The database is tested by using SQL query and checking the database from phpMyAdmin control panel. Next, the data from the database is called using PHP and SQL query to make sure the correct query is used to get the specified data as shown in Fig. 9.

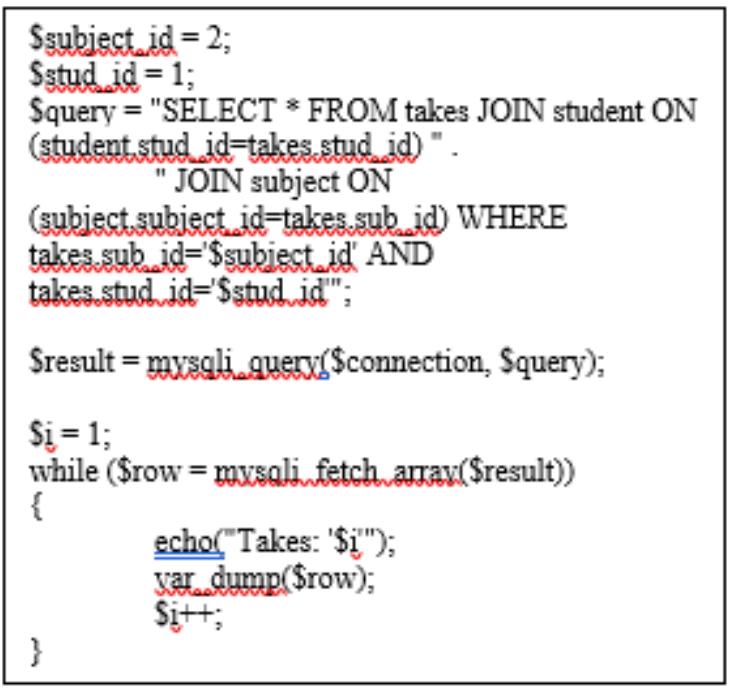

Fig. 9: PHP and SQL query testing

To display a graphical representation of the data, the design of the web page is first created based on Bootstrap framework to get the basic outline of the page. After that, chart module is inserted into the page with a dummy data. After successfully display the dummy data, real data is added from the database using PHP combine into chart script within the page. After tested with real data, more data added to the pages including current data and real time data as shown in Fig. 10.

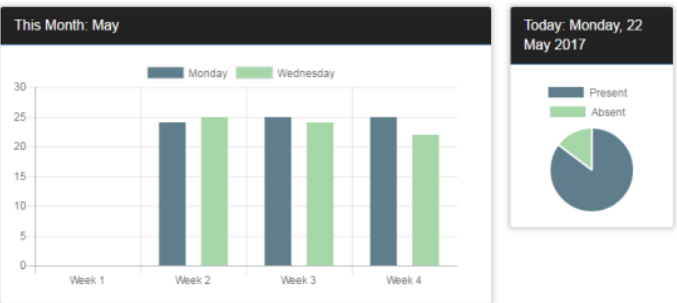

Fig. 10: PHP and SQL query testing

To give more details on the data, a list of absent student also added to the dashboard display. This list shows 2 name from the absent list and the total number of student absent in real-time. This page is linked to the list of absent student page which will show all the student absent from the particular class as in Fig. 11. The dashboard will be the main page when user logged in to the system and thus only show the summary of the overall data.

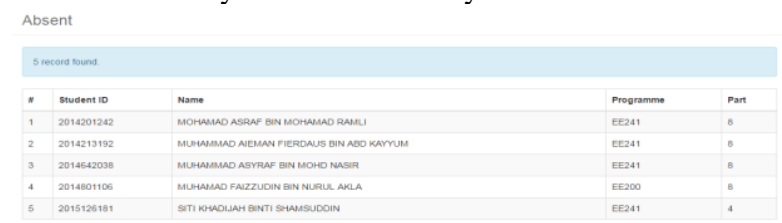

Fig. 11: Absent Student List Page 
From the summary, user can search particular student to get more info by using student id or part of the student name as a filter. User can also easily add new student to the subject database as in Fig. 12. The web view design is accompanied with a sidebar to make it easier for user to navigate between pages to get to where is required faster. Fig. 13 depicts the full web page view.

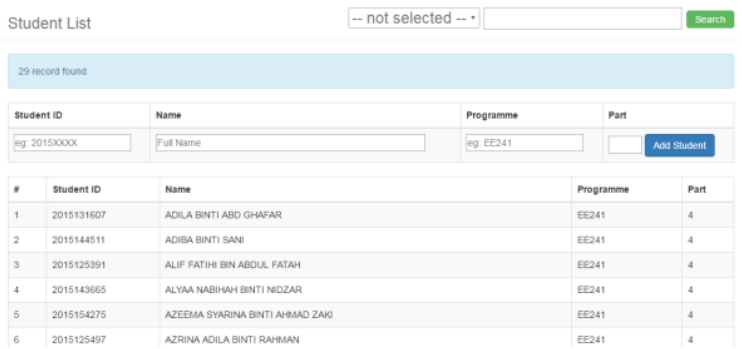

Fig. 12: Student List and Search Page

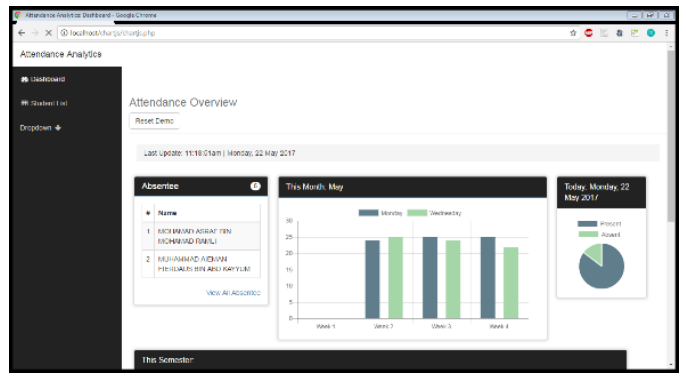

Fig. 13: Full Web Page View

\section{Conclusion}

The authors proposed the attendance system with taking the advantages the use of Internet of Things (IoT) instead of using a close system that require the use of fix hardware and software. The evolution of internet of things and data analytics will enhance and provide more information than we ever imagine with the use of readily available data that only need to be analyze to be more useful than just a $\log$ file [14]. This data could be used to provide a better service, analyze lecture quality to be improve and to inspire more student to involve in curricular and attend their classes [15]. From the proposed system, the system has successfully recorded a list of attended and absent students. Future works will focus on implementing a proper database for an institution. A face depth sensor can be used to replace the Raspberry Pi Camera and a better platform can be used with the face recognition system which is based on Python programming.

\section{Acknowledgement}

The authors would like to thank Universiti Teknologi MARA for the financial support funded by Grant number 600-IRMI/DANA 5/3/LESTARI (0175/2016).

\section{References}

[1] P. Mehta, "An Efficient Attendance Management Sytem based on Face Recognition using Matlab and Raspberry Pi 2," International Journal of Engineering Technology Science and Research IJETSR, 2016; 3(5): 71-78.

[2] H. P. LeBlanc, "The Relationship between Attendance and Grades in the College Classroom," 17th Annual Meeting of the International Academy of Business Disciplines, Pittsburg Pennsylvania, 2005; 643(210): 1-19.

[3] A. A. Mohammed and U. Jyothi Kameswari, "Web-Server based Student Attendance System using RFID Technology," International Journal of Engineering Trends and Technology (IJETT), 2013; 4(5):1559-1563

[4] S. C. Gaddam and N. V. K. Ramesh, "Attendance management and user security system's based on Eigen faces algorithm using Rasp- berry pi 2 and ethernet," Indian Journal of Science and Technology, 2016; 9(17): 8107-8112.

[5] P. S. S. Srivignessh and M. Bhaskar, "RFID and pose invariant face verification based automated classroom attendance system," International Conference on Microelectronics, Computing and Communication, MicroCom, 2016:1-6.

[6] V. O. Adeniji, M. S. Scott, and N. Phumzile, "Development of an Online Biometric- enabled Class Attendance Register System," IST-Africa 2016 Conference Proceedings Paul Cunningham and Miriam Cunningham (Eds) IIMC International Information Management Corporation, 2016: 1-8.

[7] J. Lámer, O. Kainz, and F. Jakab, "Marker based attendance systems in education process," 13th International Conference on Emerging eLearning Technologies and Applications (ICETA), 2015

[8] S. Maravi, R. Pinter, V. Vojni, V. Tumbas, and Č. Petar, "Smartphone Application for Tracking Students' Class Attendance," SISY 2016 IEEE 14th International Symposium on Intelligent Systems and Informatics August, 2016: 227-232.

[9] J. D. Sweetlin, V. Aswini, and R. Dhanusha, "Speech Based Attendance Application Register," Fifth International Conference On Recenet Trends In Information Technology, 2016:1-5.

[10] P. Wagh, "Attendance System based on Face Recognition using Eigen face and peA Algorithms," International Conference on Green Computing and Internet of Things (ICGCIoT), 2015: 303308.

[11] S. Yang, Y. Song, H. Ren, and X. Huang, "An Automated Student Attendance Tracking System Based on Voiceprint and Location," The 11th International Conference on Computer Science \& Education (ICCSE 2016), 2016: 214-219.

[12] M. Kassim, H. Mazlan, N. Zaini, and M. K. Salleh, "Web-based student attendance system using RFID technology," 2012 IEEE Control and System Graduate Research Colloquium, ICSGRC 2012 2012: 213-218.

[13] N. Chhetri, "A Comparative Analysis of Node. Js (Server-Side JavaScript)," Master Thesis St. Cloud State University, 2016: 1-79.

[14] T. Jędrzejewski, B. Trawiński, and A. Zgrzywa, "The Analysis of Data Collected by Time and Attendance Systems," Third National Conference on Scientific Data Processing Technologies, 2010: 8390.

[15] C. Science and M. Studies, "Development of a Student Attendance Management System," International Journal of Advance Research in Computer Science and Management Studies, 2014: 109-119. 